\title{
Nuevas tintas digitales para la obtención de innovadores efectos cerámicos con los que generar nuevas posibilidades y tendencias de futuro
}

\author{
GRUPO TORRECID \\ Ptda. Torreta s/n - Apdo. de Correos 18. 12110 Alcora. Castellón.
}

Este trabajo ha sido premiado con el Alfa de Oro en la Feria Internacional de Cerámica de Valencia CEVISAMA 2012

\begin{abstract}
En la presente nota técnica se recoge los aspectos más significativos del trabajo "Nuevas tintas digitales para la obtención de innovadores efectos cerámicos con los que generar nuevas posibilidades y tendencias de futuro" por el que el Grupo Torrecid obtuvo el Premio Alfa de Oro 2012 otorgado por la Sociedad Española de Cerámica y Vidrio. Para conseguir los objetivos planteados ha sido necesario trabajar en diferentes campos de actuación para cada tinta en concreto, abarcando, entre otros, aspectos como el desarrollo de fritas especiales, la selección de los disolventes más adecuados o la optimización de las composiciones para conseguir que las tintas tuvieran un comportamiento adecuado en el proceso de impresión y cumplieran todos los requisitos de estabilidad y calidad que el sector cerámico demanda.
\end{abstract}

Palabras clave: baldosas cerámicas, digital, decoración, keramcid, metalcid.

New digital ink for the obtention of innovating ceramics effects to obtain new possibilities and tendencies for the future

This technical note contains the most significant aspects of the work "New digital inks for the production of innovative ceramic effects which generate new possibilities and future trends" in which the Group Torrecid won the "Alfa de Oro 2012" given by the Spanish Society of Ceramics and Glass. To achieve the stated objectives to work in different fields of activity for each specific ink has been necessary, comprising, among other, aspects such as the development of special frits, selecting the most suitable solvents or optimizing the compositions to ensure that the inks have appropriate behaviour in the printing process and met all the requirements of stability and quality that the ceramic industry demand.

Keywords: ceramic tiles, digital, decoration, keramcid, metalcid.

\section{1.- INTRODUCCIÓN}

La tecnología de decoración digital de baldosas cerámicas $^{1,2}$ por inyección de tintas pigmentadas, que el Grupo Torrecid introdujo en el mercado en el año 2004 $\left(\mathrm{INKCID}^{\circledR}\right)$, ha supuesto una verdadera revolución dentro del sector cerámico. De hecho, su implementación a escala mundial ha tenido lugar a una gran rapidez, desbancando a otras técnicas de decoración como la serigrafía o la huecografía. Hoy en día hay cerca de 900 impresoras instaladas en todo el mundo, con las que ya se decora el $15 \%$ de la producción mundial, siendo las expectativas de continuar creciendo exponencialmente a lo largo de los próximos años.

El Grupo Torrecid, gracias a haber sido pioneros en el desarrollo de la tecnología, a la fiabilidad de las tintas que produce y a que ofrece al mercado la mejor gama cromática y la innovación con mayor valor añadido, junto con un servicio integral y personalizado, es hoy en día el $\mathrm{N}^{\mathrm{o}} 1$ en la venta de tintas pigmentadas.

Desde un punto de vista estético, hasta el momento este avance tecnológico se ha centrado totalmente en la utilización de tintas pigmentadas que aporten la parte de color relacionada con el diseño de la cerámica, por lo que a lo largo de estos años se han ido desarrollando tintas con las que abarcar una gama cromática lo más amplia posible. Sin embargo, la riqueza estética de una baldosa cerámica se ve complementada en la mayor parte de los casos mediante la aplicación de otro tipo de materiales que aportan efectos diferentes del propio color, siendo especialmente importante en las piezas de Tercer Fuego, donde los efectos metálicos y lustres adquieren una destacada relevancia.

\section{2.- OBJETIVO}

El Grupo Torrecid, siendo fiel a su estrategia de ser líderes mundiales en innovación para generar soluciones y tendencias de futuro con las que proporcionar a sus clientes las mejores ventajas competitivas y el mayor valor añadido, se planteó el objetivo de desarrollar un conjunto de tintas con las que llenar el vacío comentado en el apartado anterior y con 
las que se consiguieran los efectos cerámicos que se indican a continuación:

Tintas KERAMCID ${ }^{\circledast}$ para baldosas cerámicas de monococción:

- $\quad$ Efecto micro-relieve transparente.

- Efecto brillo sobre esmaltes mate

- Efecto nácar

- $\quad$ Efecto mate - transparente.

Tintas METALCID ${ }^{\circledR}$ para piezas cerámicas de Tercer Fuego:

- $\quad$ Efecto oro metal.

- Efecto platino metal.

- Efecto lustre.

\section{3.- DESARROLLO EXPERIMENTAL DE LAS TINTAS}

Para conseguir los objetivos planteados ha sido necesario trabajar en diferentes campos de actuación para cada tinta en concreto, abarcando, entre otros, aspectos como el desarrollo de fritas especiales, la selección de los disolventes más adecuados o la optimización de las composiciones para conseguir que las tintas tuvieran un comportamiento adecuado en el proceso de impresión y cumplieran todos los requisitos de estabilidad y calidad que el sector cerámico demanda.

\section{1.- KERAMCID ${ }^{\circledR}$ : Tintas para baldosas cerámicas de monococción.}

En las cuatro tintas desarrolladas para baldosas cerámicas de monococción (efecto micro-relieve, brillo sobre esmaltes mate, nácar y mate - transparente) el trabajo realizado se ha centrado fundamentalmente en la investigación de componentes cerámicos que proporcionaran los efectos estéticos buscados y en la búsqueda e identificación de dispersantes que garantizaran una estabilidad óptima de las tintas.

La tinta que genera el efecto micro-relieve se caracteriza principalmente por presentar una temperatura fluidez, medida por microscopía de calefacción, muy inferior a la temperatura de reblandecimiento de los esmaltes base, de forma que cuando estos comienzan a reblandecer, la tinta ya se encuentra totalmente fundida, originando las correspondientes depresiones.

En el caso de la tinta para el efecto brillo, el aspecto fundamental es disponer de una frita que no desvitrificara fases cristalinas $^{3}$ y que presentara una elevada temperatura de reblandecimiento siendo además ésta muy cercana a la temperatura de fluidez, para conseguir que las nanopartículas presentes en la tinta no fueran digeridas por los esmaltes base.

Por lo que respecta a los efectos nácar ${ }^{4} \mathrm{y}$ mate transparente hubo que recurrir a la desvitrificación de fases cristalinas de diferente naturaleza en función del efecto buscado, siendo de especial relevancia que los tamaños de los cristales generados fueran nanométricos, tal como se puede observar en las siguientes fotografías.
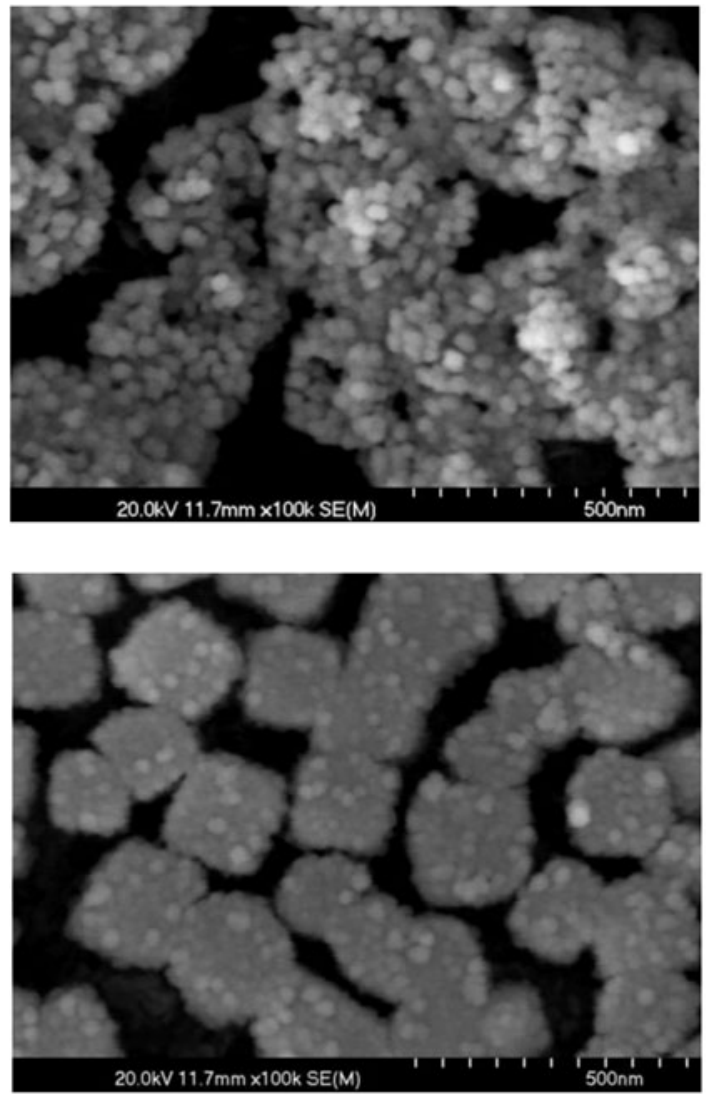

Cristales nanométricos desvitrificados en los efectos nácar y mate transparente

\section{2.- METALCID ${ }^{\circledR}$ Tintas para piezas cerámicas de Tercer Tuego.}

El Tercer Fuego representa una parte muy importante del sector cerámico, no solamente por el volumen de negocio que maneja, sino también por el valor añadido que aporta a la baldosa cerámica base y que hace que en muchos casos la cerámica sea el material elegido como recubrimiento frente a otro tipo de opciones.

Son innumerables los efectos estéticos que se ofertan dentro del campo del Tercer Fuego utilizando productos muy diferentes (granillas, vitrosas, pastas serigráficas, etc.), si bien una parte muy significativa la ocupan los efectos metálicos con oro y platino y lustres basados en Titanio ${ }^{5}$, a los cuales se les da una relevancia especial tanto por el efecto decorativo que proporcionan como por el valor que tiene el propio producto. Actualmente estos efectos metálicos y lustres se consiguen mediante aplicación serigráfica de compuestos organometálicos disueltos en disolventes difíciles de manejar y que presentan un cierto grado de toxicidad, lo que conlleva toda una serie de inconvenientes relacionados con la repetitividad de las piezas, la limpieza de los elementos que entran en contacto con las pastas serigráficas, pérdida de material de elevado coste, baja productividad, imposibilidad de decorar bajorrelieves y un largo etcétera. Todo ello hace que la utilización de la tecnología de inyección de tinta para conseguir esta tipología de efectos tenga un sentido especial en el campo del Tercer Fuego, debido a las ventajas de producto, proceso, gestión y medioambientales que proporciona y que son ampliamente conocidas. 
Por otra parte, a diferencia de las baldosas de monococción, en las cuales el esmalte sobre el que se deposita la tinta es poroso y por lo tanto absorbe la parte líquida de la misma fácilmente, en las piezas cerámicas de Tercer Fuego la superficie se encuentra vitrificada, sin que dicha parte líquida de la tinta pueda penetrar en ella, lo que entraña una dificultad adicional a la hora de conseguir una buena definición y calidad de imagen. Por lo tanto, en el desarrollo de esta tipología de tintas fue necesario llevar a cabo una investigación sobre disolventes que tuvieran la temperatura de evaporación adecuada para que una vez depositada la gota de tinta sobre la superficie vitrificada, el disolvente se evaporara lo suficientemente rápido para que la gota mantuviera su forma, pero evitando al mismo tiempo que se produjeran problemas de resecado en el cabezal que obturaran los orificios del mismo. Además, se vio que los compuestos químicos organometálicos disponibles comercialmente utilizados en las tintas serigráficas, no eran solubles en los disolventes que cumplían las características indicadas anteriormente, por lo que también fue necesario abordar el desarrollo de nuevos compuestos químicos organometálicos específicos para este tipo de aplicación.

Todo ello se tuvo que compaginar con que las tintas desarrolladas presentaran los valores apropiados de viscosidad y tensión superficial para conseguir un correcto comportamiento en el proceso de impresión, lo que dio lugar a una nueva tipología de tintas completamente distintas de las existentes hasta el momento en el mercado, tanto desde el punto de vista de formulación, como de los efectos estéticos que proporcionan.

En el caso particular del Tercer Fuego, las tintas digitales para efectos metálicos desarrolladas van a tener una especial incidencia, ya que a los aspectos estéticos que aportan se unen significativas mejoras productivas y medioambientales:

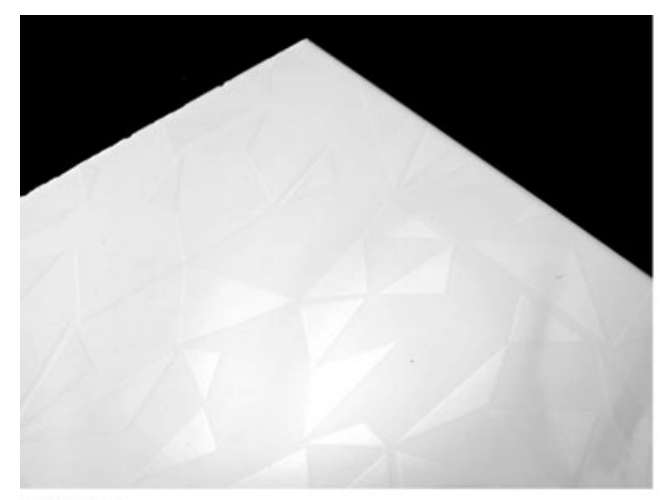

\section{PURAMIDI}

KERAMCID ${ }^{\circledR}$ : efecto nácar sobre esmalte mate

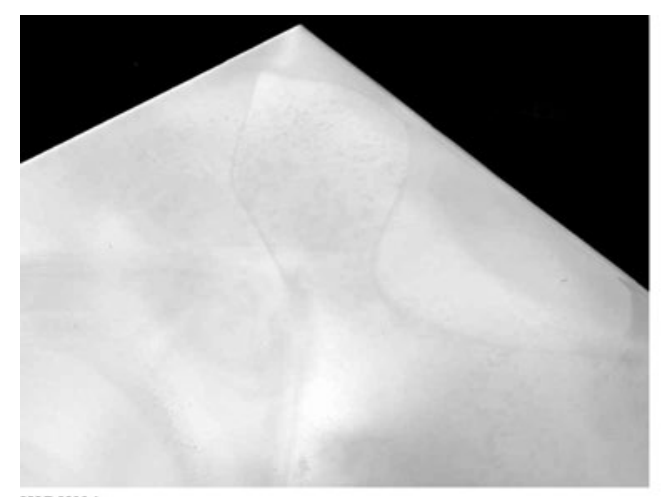

KURUNA

KERAMCID ${ }^{\oplus}$ : efecto mate - transvarente
- Eliminación de operaciones manuales.

- Decoración simultánea con diferentes tintas sin necesidad de secar previamente cada aplicación.

- Ahorro de costes al minimizar las pérdidas de compuestos de metales preciosos.

- Eliminación de disolventes con cierto grado de toxicidad.

- Mejora de la calidad de impresión, frente a las técnicas empleadas actualmente como serigrafía y pincelado.

- Decoración de piezas con relieve pronunciado, con una definición fotográfica tanto en las zonas altas como bajas del relieve.

- Rapidezy flexibilidad a la hora de desarrollar modelos.

\section{4.- DESARROLLO DE MODELOS CON EFECTOS CERÁ- MICOS}

Los efectos cerámicos obtenidos con METALCID ${ }^{\circledR}$ y $\mathrm{KERAMCID}^{\circledR}$, combinados con la amplia gama cromática proporcionada por las tintas digitales pigmentadas y las numerosas ventajas que proporciona la tecnología digital de impresión (número de piezas diferentes ilimitado, decoración de bajo-relieves, decoración hasta los bordes, elevada calidad de imagen, etc.), han permitido conseguir una gran variedad de modelos con unas características estéticas impensables hasta la fecha.

Con ello se abren nuevas posibilidades de decoración y por lo tanto se amplía la gama de producto final que ofrecer al mercado, aportando un valor añadido adicional y contribuyendo a mejorar la competitividad del sector en general.

A modo de ejemplo se incluyen a continuación fotografías de algunos de los modelos desarrollados, indicando en cada caso el efecto conseguido.

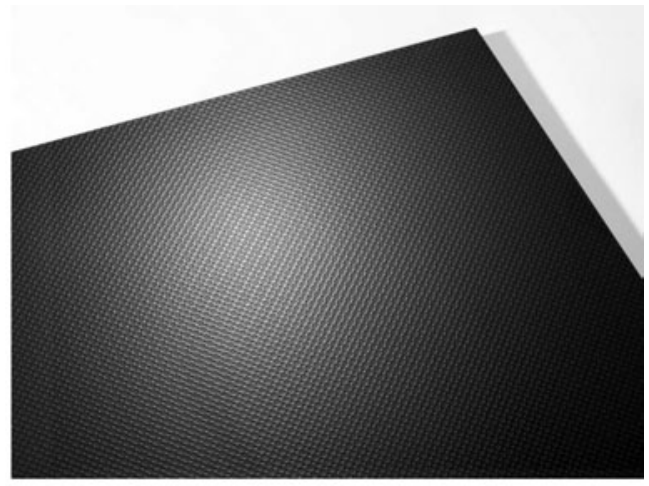

TRACIA NEGRO

KERAMCID ${ }^{\otimes}$ : micro-relieve sobre esmalte mate

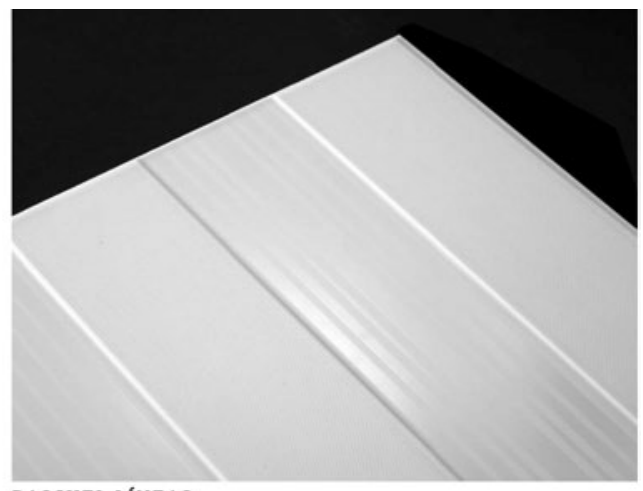

BASCHEL LINEAS

KERAMCID ${ }^{\oplus}$ : efecto brillo sobre esmalte mate combinado 


\section{5.- CONCLUSIONES}

Esta Innovación, galardonada por la SECV con el Alfa de Oro 2012, va a marcar un hito en el desarrollo de nuevos modelos dentro del sector cerámico, lo que proporcionará un posicionamiento de liderazgo de la cerámica con respecto a otro tipo de materiales y permitirá que sea un referente a la hora de marcar tendencias de futuro.

De hecho, la combinación mediante decoración digital de los efectos cerámicos desarrollados con los ya conocidos efectos cromáticos, junto con la utilización de soportes cerámicos de fino espesor (SLIMCID ${ }^{\circledR}$ ), son los aspectos que van a definir la cerámica en los años entrantes y sobre los que se va a basar su desarrollo.

Todo ello contribuirá a mejorar la competitividad del sector cerámico en general, ya que la riqueza estética que aporta esta
Innovación va a ser muy bien acogida por prescriptores como arquitectos, interioristas, etc., representando una ventaja competitiva frente a otros productos con los que la cerámica entra en competencia.

\section{BIBLIOGRAFÍA}

1.- Hutchings I. (2010) "Impresión por chorro de tinta para la decoración de baldosas: tecnología y oportunidades", QUALICER'10.

2.- I quaderni di ACIMAC (2009) "La decorazione digitale di piastrelle ceramiche".

3.- Sanmiguel F. (1998) “Influencia del vidriado base en la desvitrificación de granillas", QUALICER 00

4.- Rodrigo J.L. (1996) “Estudio de algunas de las variables que influyen sobre el brillo de vidriados obtenidos a partir de mezclas de frita y óxido de wolframio", QUALICER 94.

5.- Ruiz O. (2008) "Estudio de la capacidad de degradación fotocatalítica de vidriados cerámicos", QUALICER 08.

6.- www.secv.es

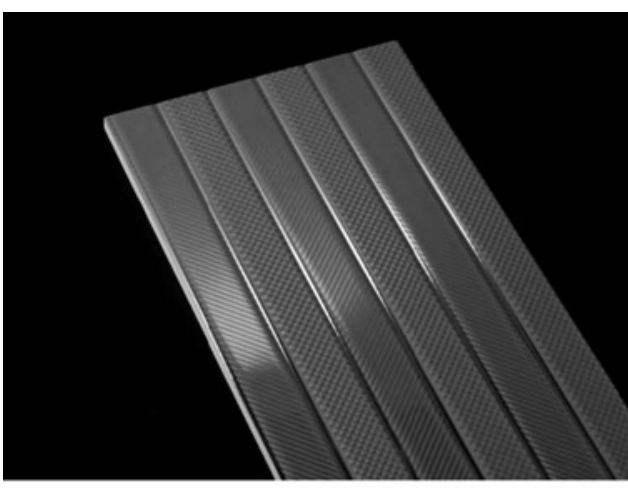

ANTARES VERDE

KERAMCID $^{\star}+$ METALCID $^{\circledast}$ : platino con micro-relieve

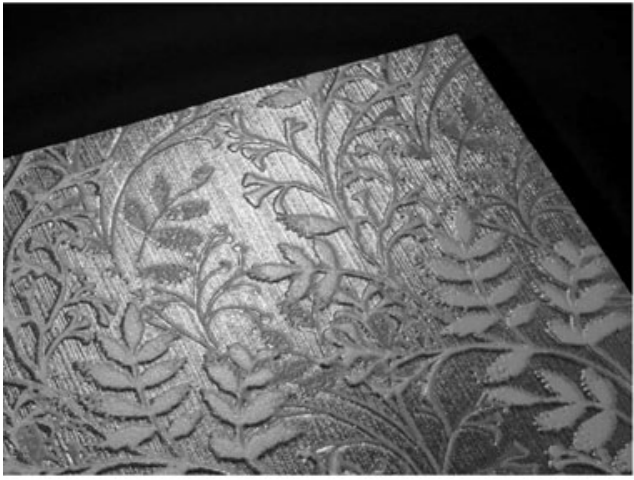

SHETLAND

METALCID ${ }^{\otimes}$ oro metal en baio-relieve

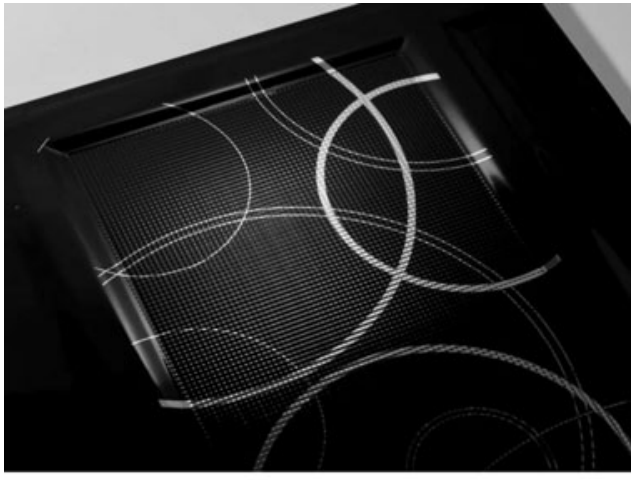

LAZZ

KERAMCID + METALCID ${ }^{\bullet}$ : oro metal sobre micro-relieve

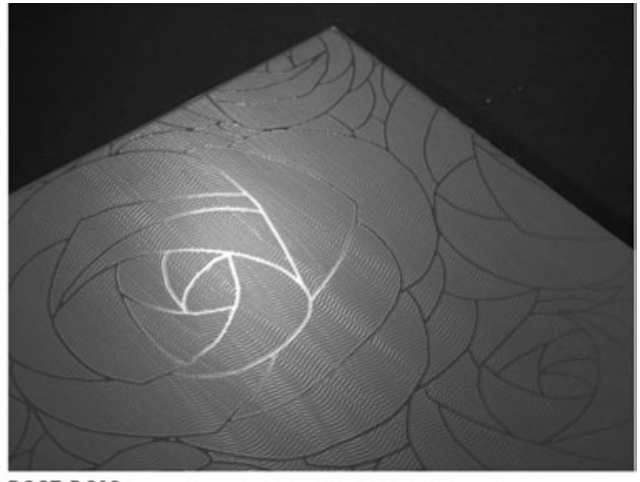

\section{BOSEROLO}

KERAMCID $^{\circledR}$ : brillo sobre esmalte mate combinado con mirrneraliave

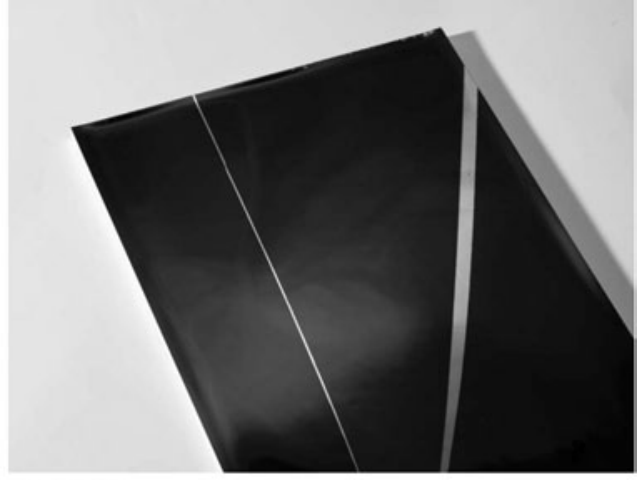

ACUARELA

METALCID $^{\star}$ : platino metal

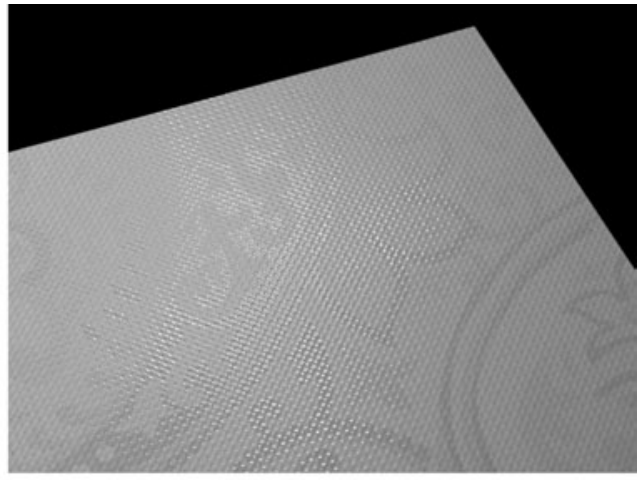

$\frac{\text { TRACIA BLANCO }}{\text { KERAMCID }^{\circledast}+\text { METALCID }^{\circledast}: \text { lustre sobre micro-relieve }}$ 